\title{
Utilidad de la cuantificación de carga viral de VIH en líquido cefalorraquídeo, a propósito de un caso
}

\author{
Catalina Gutiérrez', Leonardo Chanqueo ${ }^{1,2}$, Fernando Bernal', \\ Alejandro Ramírez², Oscar Loureiro y Patricia Vásquez'
}

\begin{abstract}
Clinical utility of HIV viral load assessment in cerebral spinal fluid, a case report
Detection of virus in cerebrospinal fluid (CSF) in HIV-infected patients with HIV viral load (VL) undetectable in plasma has been termed viral escape. These leaks may be asymptomatic from a neurological point of view, similar to plasma blips, or associated with neurological disease, with discordant VL between plasma and CSF, and may be evidence of a compartmentalization of the virus and the possibility of identifying quasispecies with mutations that confer resistance to ART. We present the case of a man with AIDS and disseminated tuberculosis who presented neurological symptomatology evidenced by headache and convulsive syndrome, who presented a discordance between plasma and CSF HIV VL; the genotypic test of the virus, obtained by lumbar puncture, identified new mutations that determined a change in ART with subsequent satisfactory evolution.

Keywords: HIV; HIV viral load; cerebrospinal fluid; viral escape.

Palabras clave: VIH; carga viral VIH; líquido cefalorraquídeo; escape viral.
\end{abstract}

\section{Introducción}

E 1 virus de inmunodeficiencia humana (VIH) se considera un virus neurotrópico que afecta el sistema nervioso central (SNC) en etapas tempranas de la infección ${ }^{1}$. La terapia anti-retroviral (TARV) ha demostrado un gran impacto, reduciendo la carga viral (CV) tanto en plasma como en líquido cefalorraquídeo (LCR), logrando niveles indetectables.

La detección de material genético (ARN viral) en LCR en pacientes en TARV con CV de VIH en plasma indetectable se ha denominado escape (blips) viral. Estos escapes pueden ser asintomáticos, similares a los blips plasmáticos, o asociados a sintomatología neurológica² ${ }^{2}$.

Presentamos el caso clínico de un paciente con infección por VIH con TARV que desarrolló un compromiso del SNC, evidenciando una discordancia entre la CV de LCR y plasma.

\section{Caso clínico}

Varón de 36 años de edad, sin antecedentes mórbidos de relevancia. Dentro de sus hábitos destacaba un tabaquismo suspendido, sin consumo de alcohol y drogas. Había sido diagnosticado con una infección por VIH en etapa SIDA por un cuadro de tres meses de evolución de fiebre asociada a tos, sudoración nocturna, compromiso del estado general, baja de peso y diarrea. Al momento del diagnóstico tenía un recuento de linfocitos CD4 132 céls/ $\mathrm{mm}^{3}$ y una CV de 3.523.602 copias $/ \mathrm{ml} \log 6,55$ (rango intervalo cuantitativo lineal: 40 a 10.000 .000 copias $/ \mathrm{ml}$ de plasma, método ABBOTT Real Time HIV-1).

El antígeno HLA-B *5701 y el estudio de co-infecciones (HBsAg, serología para VHC y toxoplasmosis) fue negativo y VDRL no reactivo. Los hemocultivos automatizados y las baciloscopias de expectoración también resultaron negativos; la radiografía de tórax y fondo de ojo fueron normales. La tomografía computarizada (TC) de tórax, abdomen y pelvis evidenció un extenso conglomerado de adenopatías retroperitoneales y mesentéricas, algunas de ellas con centro hipodenso. Se realizó una biopsia por cirugía laparoscópica, obteniendo baciloscopias de líquido peritoneal positivas para bacilos ácido-alcohol resistentes (BAAR); el cultivo de Koch fue positivo para Mycobacterium tuberculosis complex, no siendo posible realizar el estudio de susceptibilidad.

La histopatología de un linfonodo retroperitoneal, tejido hepático y médula ósea fueron compatibles con una tuberculosis, con la tinción de Ziehl Neelsen de todas las muestras positivas para BAAR.

Con el resultado de las baciloscopias positivas se inició terapia anti-tuberculosa diaria con isoniazida, rifampicina, etambutol y moxifloxacina, ajustada por insuficiencia renal. Estando en fase trisemanal del tratamiento antituberculoso, inició TARV con tenofovir/emtricitabina y raltegravir (Score CPE - Central Nervous System Penetration-effectiveness o efectividad de penetración en el SNC de anti-retrovirales $=7$ puntos).

Durante su seguimiento, al mes de inicio de TARV,
'Servicio de Medicina Interna, Unidad de Infectología. ${ }^{2}$ Laboratorio Clínico. ${ }^{3}$ Servicio de Neurología. Hospital San Juan de Dios.

Recibido: 5 de marzo de 2018 Aceptado: 12 de julio de 2018

Correspondencia a: Catalina Gutiérrez catalina.gutierrez@redsalud.gov.cl 
destacó un recuento de linfocitos CD4 78 céls $/ \mathrm{mm}^{3}$ y CV 305.702 copias/ml $\log 5,49$. Se sospechó una falla virológica por lo que se solicitó una genotipificación estándar (Gag-Pol por método ViroSeq ${ }^{\circledR}$ VIH-1), genotipo de integrasa y tropismo viral (por RPC y secuenciación) (Tabla 1). Con este resultado y estando con rifampicina, como parte de la terapia anti-tuberculosa, se cambió TARV a tenofovir, maraviroc y etravirina $($ Score $\mathrm{CPE}=$ 6 puntos).

Se hospitalizó por persistir con fiebre, cefalea y baja de peso; se controló una TC tórax, abdomen y pelvis que no demostró cambios en las adenopatías mediastínicas, retroperitoneales y perihiliares. Estando internado presentó una convulsión tónico-clónica generalizada. Se realizó una TC cerebral que informó un edema vasogénico y calcificaciones que podrían ser lesiones antiguas o tuberculomas. Se realizó una punción lumbar que dio salida a LCR turbio, con 1.280 leucocitos $/ \mathrm{mm}^{3}, 67 \%$ de PMN, proteínas 12,4 g/l, glucosa $52 \mathrm{mg} / \mathrm{dl}$ y ADA (adenosin deaminasa) 18,6 U/1 (valor normal: $7 \mathrm{U} / 1$ ) . La tinción de Gram, cultivo corriente, baciloscopias y RPC TBC GeneXpert de LCR fueron negativos. El cultivo de Koch fue negativo a los 60 días.

Por sospecha de una tuberculosis meníngea se reinició fase diaria de tratamiento, agregándose estreptomicina. Al alta hospitalaria completó 50 dosis diarias de isoniazida, rifampicina, etambutol, estreptomicina y moxifloxacina. Posteriormente, estando en fase trisemanal de terapia antituberculosa, por alteración de pruebas hepáticas, se suspendió rifampicina, manteniéndose con isoniazida, etambutol y moxifloxacina.

Se realizó una RM cerebral que evidenció múltiples focos de realce leptomeníngeo, nodulares y lineales, de distribución aleatoria fronto-parieto-temporal, sin efecto de masa, de tipo inflamatorio-infeccioso granulomatoso.

Clínicamente el paciente persistió con crisis convulsivas y cefalea. A los cinco meses del inicio de la TARV ajustada según el genotipo, se realizó un nuevo estudio de LCR, destacando una hipoglucorraquia $29 \mathrm{mg} / \mathrm{dl}$, tinta china y látex Cryptococcus negativos. Cultivo corriente, cultivo de hongos, baciloscopias, GeneXpert MTB, RPC VEB y JC fueron negativos. Se cuantificó la CV de VIH en LCR que resultó en 983.580 copias/ml log 5,99 (método ABBOTT Real Time HIV-1, $600 \mu \mathrm{L}$ LCR) y se estudió el genotipo del virus en LCR (Laboratorio de Biología Molecular de Hospital Lucio Córdova, por método ViroSeq ${ }^{\circledR}$ VIH-1). Este demostró nuevas mutaciones comparado con el estudio plasmático previo (Tabla 2). Se modificó la TARV a tenofovir, zidovudina y darunavir/ritonavir (Score $\mathrm{CPE}=8$ puntos).

En el seguimiento el paciente completó terapia antituberculosa. La CV plasmática descendió de forma progresiva (Tabla 3) hasta ser indetectable, asociado a un aumento del recuento de linfocitos CD4 a 325 céls/ $\mathrm{mm}^{3}$. Presentó mejoría del punto de vista neurológico, con ausencia de cefalea y convulsiones. Se controló un

Tabla 1. Resultado genotipo estándar, genotipo integrasa y tropismo plasmático

\begin{tabular}{|c|c|c|}
\hline Genotipo plasmático & Mutaciones & Interpretación \\
\hline Inhibidores de la transcriptasa reversa análogos de nucleosidos (NRTI) & M184V & Resistencia emtricitabina y lamivudina \\
\hline Inhibidores no nucleósidos de la transcriptasa reversa (NNRTI) & Sin mutaciones & Sin resistencia \\
\hline Inhibidores de proteasa & L10I, A71TI & Sin resistencia \\
\hline Genotipo integrasa & Y143R, V31I, S17N, D256DE & $\begin{array}{l}\text { Resistencia a raltegravir } \\
\text { Potencial resistencia a elvitegravir }\end{array}$ \\
\hline Tropismo viral & R5 & $\begin{array}{l}\text { El virus utiliza el co-receptor CCR5, es aceptable usar antago- } \\
\text { nistas del receptor CCR5 (maraviroc) }\end{array}$ \\
\hline
\end{tabular}

nistas del receptor CCR5 (maraviroc)

\begin{tabular}{|c|c|c|}
\hline Mutaciones a anti-retrovirales & Plasma & LCR \\
\hline Inhibidores de la transcriptasa reversa análogos de nucleósidos (NRTI) & M184V & Ninguna \\
\hline Inhibidores de transcriptasa reversa no análogos de nucleósidos (NNRTI) & Ninguna & K101Q, E138K, Y181C \\
\hline Inhibidores de proteasa & L10I, A71TI & L10I, A71TI \\
\hline Integrasa & Y143R, V31I, S17N, D256DE & No realizado \\
\hline Tropismo viral & R5 & No realizado \\
\hline
\end{tabular}




\begin{tabular}{|c|c|c|c|c|}
\hline Fecha & $\begin{array}{c}\text { Carga viral plasmática } \\
\text { copias } / \mathrm{ml}\end{array}$ & $\begin{array}{l}\text { Logaritmo } \\
\text { copias } / \mathrm{ml}\end{array}$ & Genotipo plasmático & Genotipo LCR \\
\hline Pre-TARV & 3.523 .602 & 6.55 & No & No \\
\hline 1 mes post TARV & 305.702 & 5.49 & $\mathrm{Si}$ & No \\
\hline Post cambio TARV según genotipo plasmático & 180.734 & 5.26 & No & $\mathrm{Si}$ \\
\hline Post cambio TARV según genotipo LCR & 1336 & 3.13 & No & No \\
\hline Seguimiento & LDL & LDL & No & No \\
\hline
\end{tabular}

nueva muestra de LCR que mostró una mejoría en los parámetros citoquímicos: glucosa $45 \mathrm{mg} / \mathrm{dl}$, proteínas 1,62 g/L, ADA 3,2 U/L y CV indetectable.

\section{Discusión}

La prevalencia del compromiso neurológico en pacientes con infección por VIH se ha estimado entre $39 \mathrm{y}$ $70 \%$, siendo su manifestación inicial en $10 \%$ de los casos ${ }^{1}$.

El VIH ingresa al SNC por vía hematógena a través de monocitos y linfocitos ${ }^{3}$; estos monocitos-macrófagos expresan moléculas neurotóxicas y citocinas proinflamatorias, que a su vez activan astrocitos y otras células. Esta activación celular y el proceso inflamatorio asociado, aumenta la permeabilidad de la membrana hemato-encefálica, con el consiguiente aumento en la llegada de monocitos y linfocitos infectados. Asimismo, existe una infección de células progenitoras neuronales, las cuales tendrían un rol importante como reservorio viral y en la formación de un compartimento separado en el SNC $\mathrm{SN}^{4,5}$.

Dentro de las manifestaciones clínicas de la afectación del SNC, existen complicaciones primarias, como trastornos neuroconductuales y deterioro neurocognitivo asociado a VIH (HAND, por su sigla en inglés) y complicaciones secundarias asociados al inmunocompromiso, principalmente infecciones oportunistas y neoplasias ${ }^{1}$.

El SNC, al actuar como reservorio del VIH, permite su replicación a pesar de la supresión viral plasmática, escapando al control anti-retroviral y facilitando el desarrollo de resistencia ${ }^{6}$.

La presencia de ARN viral de VIH en el LCR podría provenir desde el espacio perivascular o de las células infectadas en las meninges (por la baja penetración de los anti-retrovirales, por ejemplo) o bien originarse en el plasma y atravesar el plexo coroideo durante la producción de $\mathrm{LCR}^{7}$.

Se ha denominado discordancia entre plasma y LCR cuando existen diferencias entre los niveles de ARN viral de VIH entre estos compartimentos; cuando es mayor en LCR, puede resultar en la detección de variantes VIH resistentes a fármacos ${ }^{8}$. En la mayoría de los estudios se considera que existe una discordancia en los niveles de CV si es 0,5 log mayor en el LCR que en el plasma ${ }^{8,9}$.

La cuantificación del ARN de VIH en líquido LCR se ha realizado en el contexto de pacientes asintomáticos en TARV con CV plasmática indetectable, para determinar si existen escapes o blips en el SNC ${ }^{8,10-12}$; en pacientes con viremia de bajo nivel, en los cuales es complejo amplificar y genotipicar el virus ${ }^{9}$, y en pacientes con manifestaciones neurológicas ${ }^{13}$.

En pacientes en TARV sin síntomas neurológicos, Edén y cols. demostraron que $10 \%$ de los pacientes presentó ARN de VIH detectable en LCR ( 7 de 69 pacientes) con una CV media de 121 copias/ml (rango: 54-213) ${ }^{8}$. Otro estudio del mismo autor, también en pacientes asintomáticos en TARV, detectó ARN viral en LCR en $36 \%$ de ellos, con una media de 50 copias $/ \mathrm{ml}$; cuando se estudió ARN viral plasmático, 42 pacientes $(52 \%)$ tuvieron $\geq 1$ blips viral con una media de 44 copias/ml, seis tuvieron un blips plasmático y en LCR simultáneo y uno tuvo dos blips simultáneos ${ }^{10}$. Anderson y cols. demostraron en 220 pacientes asintomáticos, $12,7 \%$ de discordancia entre $\mathrm{CV}$ en plasma y $\mathrm{LCR}^{12}$, utilizando detección de $\mathrm{CV}$ tan baja como una copia/ml.

Estos hallazgos demuestran que no es infrecuente encontrar CV detectables en rangos bajos en LCR en pacientes asintomáticos, con CV suprimida en plasma.

En pacientes con ARN de VIH detectable en LCR, existiría una inmunoactivación local, evidenciada por niveles de neopterina, marcador de activación macrofágica, elevados ${ }^{11}$.

Un estudio, en pacientes con viremia de bajo nivel y asintomáticos del punto de vista neurológico, comparó la CV de LCR de 40 pacientes con controles. Se encontró discordancia entre el LCR y plasma en $18 \%$ de pacientes (7 de 40). En el grupo control, de los que tenían CV en plasma suprimida, ninguno tuvo discordancia ( $0 \mathrm{de}$ 43 pacientes $)^{9}$. Al realizar la genotipificación viral, se 
demostraron mutaciones que conferían resistencia a la TARV, llevando a un cambio de la TARV en cinco pacientes.

Por último, en un estudio de 11 pacientes con síntomas neurológicos (que consideró discordancia a $\mathrm{CV}$ en $\mathrm{LCR}>1 \log$ que en plasma o CV en LCR > 200 y CV plasmática $<50$ copias $/ \mathrm{ml}$ ), en ocho se logró realizar genotipificación en LCR, de los cuales siete tenían mutaciones. La TARV fue modificada en 10 pacientes; todos con mejoría clínica a las cuatro semanas ${ }^{13}$.

En una revisión sistemática de pacientes con medición de CV en plasma y LCR, en la cual se consideró discordante una diferencia de 0,5 log, se demostró que el SNC actuaría como un compartimento separado, ya que se evidenció discordancia en la $\mathrm{CV}$, en la presencia de mutaciones y en uso de co-receptores ${ }^{14}$.

En nuestro paciente, la medición de la $\mathrm{CV}$ de $\mathrm{VIH}$ en plasma fue 7 días previos a la CV de VIH en LCR. Pese a que no fueron simultáneas, hubo discordancia entre la CV plasmática (180.000 copias/ml, log 5,26) y la del LCR $(983.000$ copias/ml, log 5,99) con una diferencia de $0,73 \log$. Por este motivo, se realizó una genotipificación viral en el LCR que reveló nuevas mutaciones, lo que, sumado a la suspensión de rifampicina -por toxicidadpermitió optimizar el esquema anti-retroviral con una terapia más robusta que incluyó inhibidores de proteasa con potenciador.

El desarrollo de resistencia evidenciada en el estudio ulterior en LCR, podría haberse desarrollado en plasma -el paciente tenía el antecedente de mutaciones como la M184V y otras- o bien, ser atribuidas a una replicación autónoma con una evolución de resistencia independiente en el LCR. También pudiese ser explicado por la pobre penetración de los fármacos anti-retrovirales en el LCR, resultando en bajas concentraciones de las mismas.

Según el score de efectividad de penetración de los fármacos en el $\mathrm{SNC}^{15}$, estableciendo como punto de corte para definir alta penetración $\geq 6$ puntos, los esquemas anti-retrovirales de nuestro paciente siempre estuvieron sobre ese límite. Sin embargo, existen otros estudios que no han encontrado la misma correlación de penetración de los anti-retrovirales con la $\mathrm{CV}$ en $\mathrm{LCR}^{13,16}$.

La evolución posterior de nuestro paciente fue favorable, presentando CVs tanto en plasma como en LCR indetectables; el nuevo régimen de terapia anti-retroviral, que proporciona un CPE en el SNC de 8 puntos, pudiese haber jugado un rol en lograr una supresión de la $\mathrm{CV}$ en
LCR; sin embargo, no se contó con la genotipificación simultánea en plasma para corroborar esta hipótesis.

El compromiso neurológico del paciente también pudo corresponder a una manifestación del síndrome inflamatorio de reconstitución inmune $(\mathrm{SIRI})^{17}$ posterior al inicio de la TARV, apoyado por una ADA alta que hizo sospechar una TBC del SNC. Por este motivo se utilizaron corticoesteroides y se optimizó el esquema anti-tuberculoso; sin embargo, debido a la persistencia de los síntomas neurológicos y al LCR alterado, se decidió cuantificar la CV en LCR.

Si bien la técnica utilizada para la medición de CV (Abbott RealTime HIV-1) está validada sólo para el plasma, se ha reportado su uso en LCR en otros estudios publicados $^{8,18}$.

A propósito del caso clínico expuesto, nos parece que la determinación de $\mathrm{CV}$ en LCR es una herramienta útil para confirmar el compromiso neurológico por $\mathrm{VIH}$, en pacientes en TARV con respuesta adecuada de CV en plasma, habiendo descartado la co-infección por otros agentes; también podría ser considerada en pacientes con terapias de rescate por falla virológica con supresión parcial, pese a la TARV optimizada según el genotipo y adherencia adecuada.

Agradecimientos: Laboratorio Biología Molecular Hospital Lucio Córdova.

\section{Resumen}

La detección de virus en el líquido cefalorraquídeo (LCR) en pacientes infectados por VIH con carga viral (CV) indetectable en el plasma se ha denominado escape viral. Estas fugas pueden ser asintomáticas o asociadas con enfermedad neurológica. La discordancia de la carga viral de VIH entre plasma y LCR evidenciaría la presencia de distintos compartimentos del virus, con la posibilidad de identificar quasiespecies con mutaciones específicas que confieran resistencia a la TARV. Presentamos el caso clínico de un paciente con infección por VIH en etapa SIDA y una tuberculosis diseminada que presentó un cuadro neurológico manifestado por cefalea y un síndrome convulsivo, en que se encontró una discordancia entre la CV para VIH en plasma y LCR. El estudio genotípico del virus obtenido del LCR identificó nuevas mutaciones que determinaron un cambio de la TARV, con evolución posterior satisfactoria. 


\section{Referencias bibliográficas}

1.- Skiest D J. Focal neurological disease in patients with acquired immunodeficiency syndrome. Clin Infect Dis 2002; 34: 103-15.

2.- Ferretti F, Gisslen M, Cinque P, Price R W. Cerebrospinal fluid HIV escape from antiretroviral therapy. Curr HIV/AIDS Rep 2015; 12: 280-8. doi: 10.1007/s11904-0150267-7.

3.- Miller F, Afonso P V, Gessain A, Ceccaldi P E. Blood-brain barrier and retroviral infection. Virulence 2012; 3:222-9. doi: 10.4161/ viru. 19697.

4.- Letendre S. Central nervous system complications in HIV disease: HIV-associated neurocognitive disorder. Top Antivir Med 2011; 19: 137-42.

5.- Ganau M, Prisco L, Pescador D, Ganau L. Challenging new targets for CNS-HIV infection. Front Neurol 2012; 3: 43. doi: 10.3389/fneur.2012.00043.

6.- Almeida S M. Cerebrospinal fluid analysis in the HIV infection and compartmentalization of HIV in the central nervous system. Arq Neuropsiquiatr 2015; 73: 624-9. doi: 10.1590/0004-282X20150071.

7.- Rosadas C, Puccioni-Sohler M. Relevance of retrovirus quantification in cerebrospinal fluid for neurologic diagnosis. J Biomed Sci 2015; 22: 66. doi: 10.1186/s12929-015-0170-y.

8.- Edén A, Fuchs D, Hagberg L, Nilsson S,
Spudich S, Svennerholm B, et al. HIV-1 Viral escape in cerebrospinal fluid of subjects on suppressive antiretroviral treatment. J Infect Dis 2010; 202: 1819-25. doi: 10.1086/657342.

9.- Nightingale $S$, Geretti A, Beloukas A, Fisher M, Winston A, Else L, et al. Discordant CSF/plasma HIV-1 RNA in patients with unexplained low-level viraemia. J Neurovirol 2016; 22: 852-60.

10.- Edén A, Nillson S, Hagberg L, Fuchs D, Zetterberg H, Svennerholm B, et al. Asymptomatic cerebrospinal fluid HIV-1 viral blips and viral escape during antiretroviral therapy: a longitudinal study. J Infect Dis 2016; 214: $1822-5$.

11.- Dahl V, Peterson J, Fuchs D, Gisslen M, Palmer S, Price R W. Low levels of HIV-1 RNA detected in the cerebrospinal fluid after up to 10 years of suppressive therapy are associated with local immune activation. AIDS 2014; 28: 2251-8. doi: 10.1097/ QAD.0000000000000400.

12.- Anderson A M, Muñoz-Moreno J A, McClernon D R, Ellis R J, Cookson D, Clifford D B, et al. Prevalence and correlates of persistent HIV-1 RNA in cerebrospinal fluid during antiretroviral therapy. J Infect Dis 2017; 215: 105-13. doi: 10.1093/infdis/jiw505.

13.- Canestri A, Lescure F, Jaureguiberry $S$, Moulignier A, Amiel C, Marcelin A, et al. Discordance between cerebral spinal fluid and plasma HIV replication in patients with neurological symptoms who are receiving suppressive antiretroviral therapy. Clin Infect Dis 2010; 50: 773-8. doi: 10.1086/650538.

14.- Stam A J, Nijhuis M, van den Bergh W M, Wensing A M. Differential genotypic evolution of HIV-1 quasispecies in cerebrospinal fluid and plasma: a systematic review. AIDS Rev 2013; 15: 152-61.

15.- Letendre S, Marquie-Beck J, Capparelli E, Best B, Clifford D, Collier A C, et al. Validation of the CNS penetration-effectiveness rank for quantifying antiretroviral penetration into the central nervous system. Arch Neurol 2008; 65: 65-70. doi: 10.1001/archneurol.2007.31.

16.- Rawson T, Muir D, Mackie N E, Garvey L J, Everitt A, Winston A. Factors associated with cerebrospinal fluid HIV RNA in HIV infected subjects undergoing lumbar puncture examination in a clinical setting. J Infect 2012; 65: 239-45. doi: 10.1016/j.jinf.2012.04.007.

17.- Bell L C, Breen R, Miller R F, Noursadeghi M, Lipman M. Paradoxical reactions and immune reconstitution inflammatory syndrome in tuberculosis. Int J Infect Dis 2015; 32: 39-45. doi: 10.1016/j.ijid.2014.12.030

18.- Peluso M J, Ferreti F, Peterson J, Lee E, Fuchs D, Boschini A, et al. Cerebrospinal fluid HIV escape associated with progressive neurologic dysfunction in patients on antiretroviral therapy with well controlled plasma viral load. AIDS 2012; 26: 1765-74. doi: 10.1097/ QAD.0b013e328355e6b2. 